\title{
Visión de jóvenes Costarricenses, de zonas rurales, en un programa de rehabilitación, sobre el consumo de drogas
}

\author{
Ligia Murillo-Castro $^{1}$ \\ Adriana Inocenti Miasso ${ }^{2}$
}

\begin{abstract}
Este estudio cualitativo tuvo como objetivo investigar la visión de jóvenes Costarricenses, institucionalizados en dos organizaciones no gubernamentales de Costa Rica, sobre el consumo de drogas ilícitas. Se aplicó la técnica de grupo focal para que los adolescentes expresasen sus sentimientos y sus vivencias acerca del consumo de drogas ilícitas. Entre los resultados se obtuvo que la percepción de los adolescentes sobre sí mismo tiene como enfoque un proceso de cambio y de desarrollo de la identidad y experimentación que los hace tomar decisiones negativas, como es el consumo de drogas. También, se encontró que las relaciones que tienen con sus padres son conflictivas debido a que provienen de hogares disfuncionales y de comunidades marginales. Los amigos representan el apoyo a sus vicisitudes y la inducción del consumo. Se concluye que es necesario que Costa Rica modifique y cree nuevas políticas públicas para mejorar la calidad de vida de este grupo poblacional.
\end{abstract}

Descriptores: Drogas Ilícitas; Adolescente; Relaciones Familiares.

\footnotetext{
${ }^{1}$ Enfermera, Profesora, Escola de Enfermagem, Universidade de Costa Rica, San José, Costa Rica. E-mail: ligia56@gmail.com.

2 Enfermera, Doctora en Enfermería. Profesor Doctor, Escola de Enfermagem de Ribeirão Preto, Universidade de São Paulo, Centro Colaborador de la OMS para el Desarrollo de la Investigación en Enfermería, SP, Brasil. E-mail: amiasso@eerp.usp.br.
}

Correspondencia:

Adriana Inocenti Miasso

Universidade de São Paulo. Escola de Enfermagem de Ribeirão Preto

Departamento de Enfermagem Psiquiátrica e Ciências Humanas

Av. dos Bandeirantes, 3900

Bairro: Monte Alegre

CEP: 14040-902 Ribeirão Preto, SP, Brasil

E-mail: amiasso@eerp.usp.br 


\title{
Visão de jovens costarriquenhos da zona rural, em programa de reabilitação, sobre o consumo de drogas
}

Trata-se de estudo qualitativo que teve como objetivo investigar a visão de jovens costarriquenhos, institucionalizados em duas organizações não-governamentais de Costa Rica, sobre o consumo de drogas ilícitas. A técnica de grupo focal foi aplicada para que os adolescentes expressassem seus sentimentos e suas vivências sobre o processo de consumo de drogas ilícitas. Entre os resultados, obteve-se que a percepção que os adolescentes têm de si mesmos está focada em processo de mudança e de desenvolvimento da identidade e experimentação que os faz tomar decisões negativas, como o consumo de drogas. As relações que têm com seus pais são conflitantes por virem de lares disfuncionais e de comunidades marginalizadas. Os amigos representam o apoio às suas vicissitudes e à indução do consumo. Há necessidade de o Estado de Costa Rica delimitar políticas públicas para melhorar a qualidade de vida dessa população.

Descritores: Drogas Ilícitas; Adolescente; Relações Familiares.

\section{The View of Adolescents from the Rural Area of Costa Rica in Rehabilitation Programs About Drug Consumption}

\begin{abstract}
This qualitative study aimed to investigate the view of adolescents following rehabilitation programs about the consumption of illicit drugs. It was carried out in two NonGovernmental Organizations in Costa Rica. The focus group technique was applied, aiming to allow adolescents to express their feelings and experiences regarding illicit drug consumption. Results showed that the perception adolescents have of themselves is focused on a process of change and development of their identity and experimentation, which influence them in taking negative decisions like drug consumption. The relationships they have with their parents are conflicting, as they come from dysfunctional homes and marginal communities. Friends represent support for their difficulties and lead to drug consumption. The state of Costa Rica needs to define public policies to improve the lives of this population.
\end{abstract}

Descriptors: Street Drugs; Adolescent; Family Relations.

\section{Introducción}

Los problemas derivados del uso de drogas ilícitas son una de las principales preocupaciones de los países y las organizaciones internacionales. Estos problemas afectan a una proporción cada vez mayor de la población mundial(1)

En este escenario, la adolescencia, momento de grandes transformaciones en el desarrollo de los individuos, puede colocarlos en situaciones de fragilidad en relación a las influencias ambientales, es un período crucial en el ciclo de vida para el inicio del consumo de $\operatorname{drogas}^{(2)}$

Cabe señalar que la fase de experimentación con drogas suele suceder entre 10 y 12 años $^{(3)}$, y, aunque a menudo sólo se producen de forma experimental, se puede observar los patrones de comportamiento que se identifican en la edad adulta y muestran la necesidad de establecer medidas preventivas en esta etapa de desarrollo(4)

En el ámbito internacional un estudio de la Universidad de Salamanca España(5) realizado con adolescentes menciona, en sus resultados, diferencias significativas entre los sexos en las conductas de riesgo. Los muchachos presentan un mayor consumo de alcohol y de otras drogas, un mayor número de veces de estar embriagados, además de iniciar más temprano el consumo de tabaco. Mientras que las muchachas 
manifiestan un mayor consumo de tabaco que los chicos; también revelaron factores protectores como son: mayores niveles de afecto, de apoyo y supervisión de los padres.

Según la Encuesta Nacional de Juventud en Costa Rica(6), los principales problemas de los jóvenes de ambos sexos expresados por ellos son: a) en las mujeres: inseguridad ciudadana $(30,4 \%)$, falta de empleo $(30,8 \%)$, drogadicción $(11,1 \%)$, pobreza $(9,1 \%)$, corrupción política $(13,9 \%)$, alto costo de la vida $(6,1 \%) ; b)$ en los hombres: inseguridad ciudadana $(30,9 \%)$, falta de empleo $(26,6 \%)$, drogadicción $(11,1 \%)$, pobreza $(14 \%)$, corrupción política $(11,2 \%)$, alto costo de la vida $(6,1 \%)$. La cuestión de cómo se puede controlar la droga forma parte de los principales problemas de los adolescentes en el país.

Un estudio en Lima (Perú)(7) sobre el consumo de drogas en los adolescentes mostró que forma parte de una problemática de salud pública, afectando especialmente a este grupo poblacional. La opinión de ellos mostró que 53\% de los jóvenes estuvieron a favor del consumo y un 52,3\% tuvieron una percepción desfavorable a la percepción de riesgo, situación que preocupa debido a que en la actualidad los jóvenes perciben el consumo de drogas, como parte del contexto social en el cual se desenvuelven, situación cada vez más nociva para su bienestar.

En Costa Rica es apremiante conocer acerca del fenómeno de la drogodependencia, debido a que representa una preocupación para el Estado Nacional, que pretende regular el proceso de la producción, así como el tráfico y el consumo de las drogas lícitas e ilícitas. El problema de la población de niños, niñas, adolescentes y jóvenes, merece un estudio desde su propio contexto, que encuentre las condiciones o factores que influyen en el fenómeno de las drogas, que en el fondo pueden ser la causa o consecuencia de las mismas dificultades de esta población, con la finalidad de buscar constantemente respuestas para los conflictos de la vida cotidiana.

Teniendo en cuenta el contexto descrito, esta investigación tuvo como objetivo estudiar la visión de los jóvenes costarricenses, institucionalizados en dos organizaciones no gubernamentales de Costa Rica (ONGs), sobre el consumo de drogas ilícitas, permitiendo la exploración del fenómeno desde las propias voces de los jóvenes. Se cree que un estudio de esta naturaleza facilitará en el futuro la delimitación de estrategias para la conformación de programas de promoción de la salud y prevención del consumo, en los profesionales que abordan la adolescencia como grupo poblacional.

\section{Metodología}

Este estudio investigativo está fundamentado en el paradigma cualitativo con un enfoque(8) que utiliza palabras, textos, discursos y dibujos para comprender la vida social por medio de su significado y desde la perpectiva holística, pues se trata de entender el conjunto de cualidades interrelacionadas que caracterizan un determinado fenómeno.

Los sujetos del estudio estuvieran representados en dos instituciones no gubernamentales de Costa Rica que albergan adolescentes para la rehabilitación de la drogadicción. La población total estuvo constituida en estos dos centros por 30 adolescentes hombres en el Crea de Santa Lucía ubicado en la provincia de Heredia y 20 adolescentes mujeres ubicadas en el Crea de la provincia de Cartago, ambos lugares en la zona rural. Se seleccionó una muestra constituida por ocho adolescentes del sexo femenino y ocho del sexo masculino de ambas instituciones. Al respecto se consideró que el número conveniente de participantes ${ }^{(9)}$ en los grupos focales debe ser de seis a 12 participantes; la selección de este grupo de adolescentes que participaron en la investigación fue intencional y los criterios de inclusión fueron: que formasen parte de un programa de rehabilitación en estas dos instituciones para superar el consumo de drogas ilícitas en una fase de rehabilitación avanzada, esto con la finalidad de evitar la deserción de los participantes en la investigación. Los criterios éticos fue la participación de los adolescentes en forma voluntaria, firmando un consentimiento informado (firma del formulario de consentimiento) después de la aprobación del estudio por el Comité de Ética en Investigación de la Universidad de Costa Rica.

Con ambos grupos se tuvo varios encuentros para compartir sus percepciones en discusiones guiadas por la investigadora acerca de su proceso de drogadicción y los factores que incidieron en él, según la agenda diaria de encuentros. También se consultó a informantes claves que son los cuidadores de los adolescentes en ambas organizaciones no gubernamentales, constituidos por dos psicologas y seis hombres que ocupan puestos de supervisión, dirección y subdirección en las dos instituciones y que pasaron por un proceso de drogadicción y recuperación. Se aplicó un instrumento abierto con preguntas con las mismas categorías de abordaje aplicadas en los adolescentes estudiados.

Todos los encuentros de grupo con los jóvenes se registraron. Se recolectó la información paulatinamente de acuerdo a los objetivos y a las preguntas planeadas. 
Posteriormente, fue necesario diseñar una unidad de análisis programada para sistematizar la información, en base a las mismas categorías contempladas en los instrumentos. Una vez que se obtuvo el punto se saturación, se procedió al análisis de la información, de igual manera se procedió para la exploración de la información de los informantes claves o cuidadadores. Para documentar los relatos de los adolescentes e informantes se utilizo " $h$ " para el grupo de los hombres una " $\mathrm{m}$ " para las mujeres y una "ic" para los informantes claves, agregando en cada participante de cada grupo una numeración del 1 al 8 .

\section{Resultados y Discusión}

Una vez de la aplicación de los instrumentos a los adolescentes e informantes claves o cuidadores, se procedió a analizar la información, que a continuación se presenta:

\section{Percepción del adolescente de sí mismo}

La adolescencia es considerada como tránsito $(2,10)$ determinado por varios factores: la vida en familia, la experiencia escolar, el marco cultural que ha estructurado el mundo social para la puesta en práctica de normas y límites que forman la base educativa y explican la experencia de la vida comunitaria y las condiciones económicas y políticas que imperan en la generación que pasa por la experiencia de ser adolescente - momento socio histórico. La vida social de la persona adolescente representa un grupo que ha sido considerado como diferente, afrontando las críticas y dificultades del medio social en que vive. Para algunos adolescentes su sentir es expresado de la siguiente manera:

... me gusta vacilar, no me gusta expresar, me gusta experimentar, me gusta la velocidad como andar en moto, me gustan los deportes extremos. (m.4)

... nosotros somos personas que tomamos decisiones pero mal encaminadas, al final cuando nos sentimos perdidos es cuando tenemos una toma de decisiones pero para buscar ayuda, por algo llegamos a un lugar como este para rehabilitarnos. (h.8)

... para admitir que tenemos un problema con las drogas, se nos hace muy difícil, nos falta confianza para admitir, sino hasta que cometemos delincuencias y caemos en algo muy grave. (h 6)

Para los jóvenes la identidad es un proceso que deben experimentar cuando empiezan a definirse en el papel de ser hombre o mujer dentro de la sociedad - es la adolescencia; por lo tanto es una etapa del sentido creciente del(11) yo donde él o ella tratan de ajustarse al medio en el cual se desenvuelven como personas, con sus propias crisis.

En el grupo social de los adolescentes que consumen drogas, a través de sus relatos, muestran que los hombres describieron una identidad negativa. El mismo autor ${ }^{(11)}$ muestra como el adolescente es transformado en un nueva persona, que lo hace cometer actos amorales, prohibidos o de riesgo que lo convierte en un extraño para los miembros de su propia familia, confrontándolo además con su yo ideal, que ocasiona como consecuencia un choque con su propio yo, con un yo real. En este caso particular, reconocen dentro de sí mismos que tienen un problema con la adicción a las drogas ilícitas, que necesitan ayuda para resolverlo, tomando conciencia que no lo pueden hacer solos y es ese el momento en que se acercan a instituciones, como las organizaciones no gubernamentales, para iniciar un proceso de rehabilitación.

Diversos factores giran alrededor de la adolescencia, sin embargo se debe establecer la diferencia y respetar las individualidades. El análisis de cada realidad marca la diferencia en la conceptualización de un grupo poblacional que cada vez ofrece más cambios, que al mismo tiempo está impregnado por el impacto de cada época en particular, actualmente la globalización de los mercados, el consumismo, el uso de la tecnología cada vez más sofisticada, el mundo de la moda, que simbolizan para nuestros adolescentes un modelo a veces imitado por muchos de ellos y que además tienen mensajes ocultos, como el uso de sustancias lícitas e ilícitas, para sentir creatividad, movimiento. Este tipo transmisión de comunicación distorsionada encanta al adolescente y lo conduce por caminos cargados de riesgo, que luego le irán a ocasionar consecuencias muy nefastas.

\section{Sentimientos que tienen los adolescentes en el inicio del consumo de drogas ilícitas}

Las sensaciones y los sentimientos que sufren y expresan los adolescentes cuando inician el proceso de consumo de las drogas ilícitas son las siguientes:

... sentía soledad y miedo, muchos vacíos, sufría de depresiones, no valía nada. (m.3)

.. mis padres no me dieron importancia, sentía vacíos desde la niñez, mi papá siempre estaba borracho, entonces busque los amigos negativos. (m.1)

... sentía odio, rencor, vacíos, yo entonces empecé a buscar gente negativa, por los problemas de mi familia y porque mis papás se separaron. (m.8)

... mi mamá alcohólica y padrastro también, él agredía a mi mamá, me sentía muy sola, busqué entonces personas negativas, me sentía menos que los demás. (m.2) 
... mi papá alcohólico por años, tengo cinco hermanas, quería morirme, estaba harta, me sentía como si nunca había tenido padres. (h.4)

Las adolescentes mujeres como se pude observar son más expresivas que los hombres, en sus relatos. Existen sentimientos en ambos sexos de minusvalía, de baja autoestima, problemas familiares, soledad y desesperanza. La droga representa para los jóvenes una salida equivocada para aminorar las situaciones conflictivas que enfrentan.

La edad promedio de este grupo de adolescentes, está ubicada en la adolescencia media la cual según la literatura(12) es la etapa de la vida donde él o ella tienen los sentimientos de experimentación y omnipotencia, esto es lo que favorece en este grupo poblacional la toma de decisiones equivocadas en el inicio del consumo de drogas lícitas; inicia el consumo de drogas ilícitas primero, como un mecanismo para disminuir sus emociones negativos y luego para buscar nuevas experiencias y mayores sensaciones. Esto hace de la adolescencia una etapa de vulnerabilidad al comportamiento social, según lo refiere un estudio de la Universidad de Madrid(13). Así de la misma manera lo expresan los adolescentes:

... empecé fumando cigarrillo a los 11 años y tomando licor, luego pase al consumo de marihuana. (m.5)

... empecé fumando marihuana porque siempre veía a mi abuelito fumar, de ahí aprendí. (m.1)

... inicie fumando luego decidí tomar licor y después pase a la marihuana y cuando ya sentí que necesitaba un efecto más grande probé todas las demás drogas como el crack. (h.2)

En esta fase el adolescente no tiene conciencia de lo realmente que está adquiriendo con el consumo de drogas ilícitas, una vez que comienza no puede parar, es agobiado por la conducta adictiva y por el deseo de buscar más efecto en ese proceso y por disminuir sus aflicciones. Un estudio sobre la adolescencia muestra las relaciones de los jóvenes con conductas adictivas y el asilamiento social, además de las relaciones conflictivas con sus padres y el desacuerdo escolar(14). De igual manera encontramos en esta investigación, que los sentimientos de inestabilidad de los jóvenes, en el inicio del consumo, son siempre de conflicto, siendo más evidente dentro de la relación familiar, iniciando de esa forma el consumo de drogas buscando alivio para esos sentimientos.

\section{La relación de los adolescentes con sus padres}

Uno de los aspectos expresados por los adolescentes, cuando inician el consumo de las drogas, son los conflictos que surgen en la relación cotidiana familiar. Expresiones que a continuación se exponen:
... habían muchos problemas en mi familia, mi mamá me agredió mucho. Me golpeaba. (m.6)

... mi papá me encontró probando fumar, más me obligó a fumar un cigarro completo para que probará, luego él y yo fumamos y bebimos licor juntos. (m.1)

... mi papá y mi mamá vendían drogas, siempre habían muchos conflictos en el hogar desde niño pasé viendo la policía entrar y salir de mi casa, toda la familia es narcotraficante de la droga. ( h.1)

... Las relaciones muy conflictivas entre mis padres $y$ entre toda la familia, siempre un ambiente rodeado de muchos problemas. (h.3)

... familia disfuncional debido a que los padres la mayoría del tiempo han socavado la autoestima de los adolescentes. (ic.8)

... en cuanto a los adolescentes que consumen drogas, la relación que tiene con sus padres es distante, fría, rebelde. (ic.4)

La familia es la unidad social históricamente reconocida a través de las distintas épocas; ésta en la etapa de la adolescencia representa para los jóvenes un factor protector porque los padres deben asumir el papel de brindar y satisfacer las necesidades básicas, así como favorecer el desarrollo psicosocial del adolescente mediante un vinculo afectivo, que une a los miembros en la dinámica familiar. La familia ha sido definida por el modelo de sistemas(15) como un sistema vivo cuyos miembros interactúan entre sí y cuando una situación particular afecta a uno de sus miembros tiene influencia sobre el resto de los integrantes.

La familia se constituye en un constructor social; cada país y cada sociedad tiene su forma particular, desarrollándose dentro del marco de su propia dinámica. En el grupo estudiado las familias de los adolescentes están constituidas por mujeres jefas del hogar, debido a que sus compañeros las abandonaron a su suerte y por lo tanto el padre no asumió la responsabilidad paterna de los hijos. Como consecuencia, ellas han tenido que asumir trabajos informales donde obtienen un recurso económico muy limitado para el sustento de sus hijos, de manera que la mayoría de estos jóvenes proviene de hogares pobres. En algunos casos, en menor grado, están presentes los dos padres pero las relaciones son sumamente conflictivas, situación que ha sido expresado por los jóvenes y cuidadores.

Otro componente importante de la dinámica familiar de este grupo poblacional es que algunas de ellas, son consumidoras y traficantes de drogas ilícitas, situación que hace vivir a los adolescentes este proceso desde edades muy tempranas, siendo los mismos padres los que los inducen a los jóvenes a asumir este flagelo 
humano. La pobreza, es una característica de las familias de los adolescentes. Un estudio sobre la percepciones de la familia con bajos ingresos apunta ${ }^{(16)}$ el predominio de mujeres en el cumplimiento del papel de jefe de familia, también el tráfico y consumo de drogas en las familias, ocasionando como resultado, muy nefasto, la agresión que forma parte de su entorno lo que suscita la disfuncionalidad familiar.

\section{La relación de los adolescentes con sus amigos}

Los amigos representan para los jóvenes como auténticamente relevante en la etapa de la adolescencia media. El apoyo incondicional de los pares forma parte de una subcultura creada por ellos mismos, de acuerdo a sus valores, principios, normas, forma de pensar, de vestirse, de gustos y preferencias que son compartidas en común. Tienen además su propia forma de comunicarse, con sus códigos y símbolos, creados por el propio grupo social. En este proceso de definición de identidad, de búsqueda y de cambio, los amigos son muy importantes para los adolescentes, en algunas situaciones a veces más importantes que su propia familia, en particular en el proceso de consumo de drogas ilícitas y de rehabilitación. La relación de amistad de los adolescentes con sus amigos o pares son las siguientes:

... mis amigos me veían tan golpeada que me ofrecían la droga, inicie a los 12años. (m.5)

... mis amigos influyeron mucho, quería ser aceptada por ellos y para eso tenía que consumir. (m.1)

... los amigos influyen porque uno está muy confundido. (h.6)

... algunos de mis amigos eran buenos, pero yo les fallé les hice mucho daño, al final me quedé sin ellos, la droga te consume poco a poco. (h.8)

... el grupo de amigos ejerce una gran influencia para hacer o no algo, dígase competir en una carrera, empezar a fumar o beber e inclusive a desobedecer a los padres. (ic.7)

Tal y como fue expresado por los adolescentes y los informantes claves, el joven en su grupo de iguales, visualiza a los amigos ${ }^{(17)}$ como el inductor de seguridad, el árbitro de aceptación o rechazo y el indicador de lo permitido y lo prohibido, de lo aceptable y lo inaceptable. La relación de la amistad no siempre es negativa, los grupos le ofrecen al adolescente(14) del mismo modo: afecto, comprensión y solidaridad que no consiguen con sus padres y otros adultos debido a las relaciones conflictivas, pero que sin embargo caen en el fenómeno de las drogas como una nueva exploración y alivio a sus vicisitudes cotidianos.

\section{El entorno de la escuela y colegio en los adolescentes}

El principal problema que se presenta en la educación tanto primaria como secundaría en Costa Rica, con el adolescente, una vez que se identifica el consumo de drogas ilícitas, es el problema de la estigmatización; por otro lado, no se hacen esfuerzos para iniciar nuevas exploraciones de apoyo y tratamiento en la rehabilitación del joven, más bien al contrario, se convierte en una lucha de poder en la búsqueda de culpables entre la escuela, colegio y la familia, que en definitiva, no favorece la indagación de nuevas soluciones. Se ha comprobado dentro de algunas escuelas el tráfico de drogas:

... dentro de la escuela hay consumo e intercambio de sustancias y no si no se da la intervención oportuna, evidentemente se convierte en un problema. (ic.7)

... me sacaron del colegio, mis notas eran malas. (m.6)

... engañaba a los profesores para salirme del colegio y drogarme. (m.2)

... es impresionante en el colegio la entrada a séptimo como se relaciona con el inicio del consumo de drogas, ese cambio es muy drástico y algunos no sobreviven a él sin marcas. (i.c5)

Los adolescentes se convierten en infractores dentro de la escuela, como un proceso que va creciendo en complejidad y que como resultado, la escuela termina expulsando al adolescente o el estudiante por iniciativa propia abandona la escuela.

... el colegio lo dejé, sacaba buenas notas cuando no consumía, pero luego inicie mi consumo de droga y ya no pude parar, ni siquiera iba al colegio mentía en mi casa. (h.6)

... tuve muchos problemas en la escuela, empecé el vandalismo, rayaba las paredes. (m.8)

En el colegio ya ni la directora me hacía caso, yo era un caso perdido, me negaron la entrada al colegio. (m.3)

Algunos otros datos que apuntan el nivel de escolaridad del grupo de adolescentes; entre los 8 hombres, 7 tienen la primaria incompleta y solamente 1 1legó a la secundaria incompleta; de las 8 mujeres, solamente 1 tenía primaria incompleta y las 7 restantes tuvieron la secundaria incompleta. Independientemente de los factores que determinaron la interrupción de sus estudios, tanto hombres como mujeres, en el contexto costarricense, se consideran y son valorados como personas que han fracasado en su proceso educativo, o desde la dimensión social, son excluidos del sistema que exige y demanda estudiantes con estudios de bachillerato concluidos. Otra consideración digna de ser mencionada se relaciona con los distintos niveles educativos, en donde las mujeres y los hombres fracasan, en la primaria 
lo hacen los hombres, mientras que en la secundaria son las mujeres.

Se constata que los adolescentes fracasan en el sistema educativo y en consecuencia son excluidos por el mismo sistema, una situación que no resuelve la problemática del fenómeno de las drogas, pero que en definitiva lo conduce más hacia el fenómeno de la adicción que al camino de la rehabilitación.

\section{El ambiente de la comunidad y la influencia en el consumo de drogas ilícitas en el adolescente}

La comunidad es el entorno en donde conviven los adolescentes con sus familias. La coexistencia de numerosos grupos sociales con disfuncionalidad en la dinámica familiar, se corresponde con la comunidad en forma recíproca, el entorno de las familias conflictivas influye sobre la comunidad y a su vez una comunidad enferma afecta a las familias en forma continuada. La problemática en el fenómeno de las drogas, trae consigo otros problemas sociales como la coexistencia de la prostitución, enunciada más por las mujeres adolescentes que por los hombres; asimismo, en ambos sexos se produce como resultado la delincuencia, roban y cometen delitos para mantenerse en el ciclo del consumo, ocasionando como resultado la desintegración social, expresado por los sujetos de la investigación de la siguiente forma:

... yo vivía en el centro de la droga, en los barrios Hatillo1, 15 de setiembre, San Sebastián, son lugares donde aparece la droga por todo lado, la policía no entra a estas comunidades, no hay una ley que impida el consumo ni la venta. (h.1)

... vivo en Puntarenas ahí está el mar y el estero, los barcos entran y traen la droga, en mi barrio abunda la droga, la policía se vende es parte de los grupos de narcotraficantes, cuando hay operativos, ellos avisan a los narcos para que estén preparados. (h.3)

... empecé consumiendo marihuana en mi casa, siempre anduve en la calle, luego pasé a la cocaína, siempre estuve en la calle me prostituía y robaba, desciendo de una familia que trafica y consume drogas. (m.8)

... si nosotros nacemos en un ambiente de drogas, prostitución uno ve eso normal y empieza a practicarlo. (ic.6)

El acceso a las drogas se facilita en las comunidades en que viven los adolescentes, vive en un ambiente de pobreza, se ve limitado de recursos y lugares que les permitan tener un estilo de vida más sano, practicar actividades físicas, y formas de recreación, entre otras. En consecuencia la droga se convierte en el primer elemento del medio ambiente comunitario para estos jóvenes; ellos conviven con ello, es un medio donde se facilita tanto el consumo como el tráfico de esas sustancias ilícitas.

... la comunidad donde vivo es una área rural, todo el mundo consumía, es un lugar precario, todas las personas consumen hasta los niños, adultos y adultos mayores. (m.3)

... la comunidad donde vivimos es precaria es un ambiente malo, todas las personas viven de vender la droga y también la consumen. (h.5)

... ambiente de la pobreza, de estatus social bajo, en donde no hay lugares de recreación, no practican algún deporte, este ambiente es la causa para consumir drogas. (ic.4)

... si un ambiente es muy pesado o muy corrupto hace de un adolescente un posible adicto o antisocial. (ic.3)

Desde esta perspectiva, las condiciones de pobreza en algunas comunidades, facilita para que socialmente se internacionalice el consumo y tráfico de las drogas ilícitas, avanzando y progresando sin ningún control del Estado y convirtiéndose en un fenómeno que es parte de un subsistema para muchas familias ${ }^{(14)}$, aspecto que necesita ser retomado por el ámbito nacional e internacional. Actualmente, debido a la drogodependencia, muchas colectividades en el contexto local de cada país, tienen sufrimiento, desolación y muertes, resultados que bajo un marco preventivo y de intervención y de voluntad política podrían evitarse.

\section{Consideraciones finales}

La adolescencia es un periodo de transformación, en el cual existe una mayor vulnerabilidad en el cambio social a los adolescentes, los que buscan constantemente una identidad propia, situación que puede favorecer la adopción de conductas de riesgo, como es el consumo de drogas ilícitas.

La globalización de los mercados y el consumismo, en la época posmoderna, traen como consecuencia para este grupo social de adolescentes, cambios y consecuencias que le aportan una visión de mundo; las innovaciones particulares del contexto universal que contienen mensajes ocultos, traen para este grupo poblacional situaciones de riesgo, como el sexo sin protección y el uso y abuso de sustancias lícitas e ilícitas. En este contexto, la familia ha sido considerada socialmente e históricamente como un mecanismo fundamental en la vida del ser humano y de las sociedades, sin embargo se evidencia a través de las expresiones de los adolescentes estudiados, que existen disfuncionalidades en la dinámica familiar, por ejemplo: pobreza, agresión, hogares constituidos por un sólo miembro con la mujer como jefa del hogar; factores que son de riesgo para los 
jóvenes, ya que quedan expuestos a las amistades de los amigos, que algunas veces incentivan la inclinación para la adopción de la drogadicción, como única salida a la solución de sus visitudes.

Además, la educación en la sociedad Costarricense excluye al joven consumidor de drogas ilícitas del sistema, debido a su bajo rendimeinto escolar, así como a su comportamiento delictivo, consecuencia que ocasiona un sentimiento de impotencia, impidiendo que los jóvenes se alejen de las drogas, y puedan recuperarse de su proceso de adicción y logren concluir sus proyectos para optimizar su calidad de vida y éxito social.

Todos estos hallazgos sugieren la necesidad de que Costa Rica examine e cree nuevas políticas públicas con el objetivo de vigilar y exigir el cumplimiento de los derechos de la niñez, adolescencia y juventud en el país; así, se podrá operacionalizar una toma de decisiones, para mejorar la calidad de vida de este grupo poblacional.

\section{Agradecimientos}

Agradecemos a la Comisión Interamericana para el Control del Abuso de Drogas/CICAD de la Secretaria de Seguridad Multidimensional/SSM de la Organización de los Estados Americanos/OEA, la Secretaria Nacional de Políticas sobre Drogas/SENAD do Gabinete de Seguridad Institucional/Brasil, la Escuela de Enfermería de Ribeirao Preto de la Universidad de Sao Paulo y Centro Colaborador de la Organización Mundial de la Salud para el Desarrollo de la Investigación en Enfermería, la población representada en los estudios de investigación, bien como a las autoridades de las universidades representadas por los participantes del Programa En-Line de Especialización en Investigación sobre el Fenómeno de las Drogas, periodos 2006, 2007, 2008 y 2009.

\section{Referencias}

1. Silva J, Ventura CAA, Costa OM, Douat CM, Albarracín E, Gonzalo D, et al. Illicit drug use in seven Latin American countries: critical perspectives of families and familiars. Rev. Latino-Am. Enfermagem. 2009;17(spe):763-9.

2. Schenker M, Minayo MCS. Fatores de risco e de proteção para o uso de drogas na adolescência. Ciência Saúde Colet. 2005;10(3):707-17.

3. Alavarse GMA, Carvalho MDB. Álcool e adolescência: o perfil de consumidores de um município do norte do Paraná. Esc Anna Nery Rev Enferm. 2006;10(3):408-16.
4. Tavares BF, Beria JU, Lima MS. Prevalência do uso de drogas e desempenho escolar entre adolescentes. Rev Saude Publica. 2001;35(2):150-8.

5. Vergeles MR, Martín AH, Martín AF, Alvarez JLM. Consumo de drogas en la adolescencia: Importancia del afecto y la supervisón parental. Psicothema. 2003;15(2):161-6.

6. Consejo Nacional de la Política Pública de la Persona Joven. Encuesta Nacional de Juventud, San José, Costa Rica: Fondo de Población de las Naciones Unidas, UNFPA, 2008. 136 p. [citado 2008 fev. 11]. Disponível em: http://cpj.go.cr/docs/encuesta/informe-encuesta.pdf.

7. Gil HLB, Mello DF, Ferriani MGC, Silva MAI. Opiniones de los adolescentes escolares sobre consumo de drogas: un estudio de caso en Lima, Perú. Rev. Latino-Am. Enfermagem. 2008;16(esp):551-7.

8. Mejía J. Sobre investigación cualitativa. Nuevos conceptos y campos de desarrollo. Rev Invest Soc. 2004; (13):277-99.

9. Aigneren M. Centro Centroamericano de población. Biblioteca virtual de población. Rev Electrónica. $2000 ; 7: 143$.

10. Scivoletto S. A adolescência. In: Fleitlich-Bilyk B, Andrade, ER, Scivoletto S, Pinzon VD. Saúde mental do jovem brasileiro. São Paulo: Editora Inteligente; 2004. $176 \mathrm{p}$.

11. Amara J. Desarrollo de la identidad. México: Asociación Mexicana de Pediatría. Graw-Hill M; 2000.

12. Dulanto E. Desarrollo psicosocial Normal. Asociación Mexicana de Pediatría. Interamericana $\mathrm{M}-\mathrm{H}$, editor. Mexico; 2000.

13. García KS, Costa Junior ML. Conducta Antisocial y consumo de alcohol en adolescentes escolares. Rev. Latino-Am. Enfermagem. 2008;16(2):299-305.

14. Guzmán FF, Pedrão LJ. Factores de riesgo personales e interpersonales en el consumo de drogas ilícitas en adolescentes y jóvenes marginales de bandas juveniles. Rev. Latino-Am. Enfermagem. 2008;16(3):368.

15. Levetón E. El adolescente en crisis su apoyo en terapia familiar. Santiago: Editorial Pax; 1987.

16. Reinaldo AMS, Pillon SC. Repercusiones del alcoholismo en las relaciones familiares: estudio de caso acerca del tema. Rev. Latino-Am. Enfermagem. 2008;16(spe):529-34.

17. Martínez JL, Fuentes A, Ramos M, Hernández A. Consumo de drogas en la adolescencia: Importancia del afecto y la supervisión parental. Universidad de Salamanca. Psicothema. 2003;15(2):161-6. 